\title{
A numerical evaluation of various damage models for thermoplastic composite materials subjected to quasi-static tensile loading
}

\author{
Ioannis Sioutis ${ }^{l}$, and Konstantinos Tserpes $^{l^{*}}$ \\ ${ }^{1}$ Laboratory of Technology \& Strength of Materials, Department of Mechanical Engineering \& \\ Aeronautics, University of Patras, Patras 26500, Greece
}

\begin{abstract}
In the present work, damage propagation in thermoplastic composite laminates subjected to quasi-static tensile loading was numerically simulated using different damage models. The simulation was performed using the commercial finite element suite LS-Dyna, where various material models were evaluated based on their ability to simulate the thermoplastic composite behavior. More specifically, material models based on Tsai-Wu failure criteria (MAT_055), progressive damage modeling (MAT_162), micromechanical material modeling (MAT_215) and continuum damage modeling (MAT_261) were implemented, comparing the output failure strength and load - displacement curve with experimental data available in the literature. The approach of damage evolution and failure type for each model was taken under consideration as well. Overall, the progressive damage model (MAT_162) presented the most accurate prediction of the material's failure behavior.
\end{abstract}

Keywords: Thermoplastic composites, Finite element analysis, Damage models, Damage propagation, Tensile loading

\section{Introduction}

The use of thermoset matrix composite materials in the aerospace industry has been growing the past decades. The advantages over their metallic counterparts include superior strengthto-weight ratio and adjustable mechanical properties depending on their application [1]. Their complexity in microstructural level leads to difficulties in mechanical response prediction. Thus, they have been the main subject of study for many researchers. Numerous works have been focused on experiments and numerical analyses of thermoset composites.

The recent need for more environmentally friendly materials has turned the attention to thermoplastic matrix composite materials. Apart from their recyclability, thermoplastics present other advantages as well. Being thermally reformable, they can be welded together avoiding the use of mechanical fasteners or adhesives, offering potential in minimizing manufacturing times[2,3]. Studies have also shown that their fracture toughness and impact resistance is higher than thermosets[4,5].

\footnotetext{
* Corresponding author: kitserpes@upatras.gr
} 
Comparing thermoset and thermoplastic matrix composites, crucial differences can be observed in their mechanical response and failure mechanisms. Higher fracture toughness and failure strain is presented in thermoplastics, thus suppressing the crack initiation and propagation in contrast to the more brittle thermosets. M. Mohd Tahir et al.[6] conducted a comparative study between epoxy and PA6 matrix quasi-isotropic laminates failure behavior under quasi-static tension. Generally, they found that in the thermoset composite prior to the final rupture of fibers, matrix cracking and delamination between $45^{\circ}$ and $90^{\circ}$ plies occurred until $70 \%$ of the total load and delamination between $0^{\circ}$ and $45^{\circ}$ plies took place above that load percentage. On the contrary, the thermoplastic laminate didn't present delamination, but the failure came up from matrix cracking propagation.

In contrast to thermosets, works on thermoplastic composites are by far more limited in the literature, due to the recently developed interest. Even though experimental studies can be found, numerical works based on finite element analyses are not common yet. Considering their differences in mechanical response to thermoset composite materials, new finite element models should be developed to simulate their behavior.

In the present study, four different LS-Dyna material damage models were investigated for thermoplastic composites under quasi-static tension. Their ability to capture the damage propagation as well as the total response of a thermoplastic matrix laminate was evaluated in comparison to experimental data available in the literature. Each damage model is based on different failure theories. Namely, Tsai-Wu failure criteria (MAT_055), progressive damage modeling (MAT_162), micromechanical material modeling (MAT_215) and continuum damage modeling (MAT_261) [7].

Overall, this work aims at the establishment of an accurate simulation technique for thermoplastic matrix composite materials, not only considering their mechanical response but their micromechanical behavior as well. This study is a part of a European project in the framework of "Clean Sky 2" with the abbreviated name TORNADO "Innovative disbond arrest features for long thermoplastic welded joints". The objective of the project is the development, testing and analysis of crack stopping elements integrated within the thermoplastic welded aircraft stiffeners and skin. As a preliminary study, it is considered crucial to simulate the damage behavior of the welded elements in order to predict failure, in case of crack branching outside of the weldline.

\section{Finite element modeling}

The finite element model of the thermoplastic laminate was developed in LS-Dyna commercial simulation software package. Firstly, the geometrical model of the tensile loading specimen was created with dimensions $130 \mathrm{~mm} \times 25 \mathrm{~mm} \times 1.12 \mathrm{~mm}$. The thermoplastic composite was stacked with 8 unidirectional plies following a $\left[45^{\mathrm{O}} / 0^{\mathrm{O}} /-45^{\mathrm{O}} /\right.$ $\left.90^{\circ}\right]_{\text {s lay-up. }}$

The specimen model was discretized with constant stress 8-noded solid elements (ELFORM 1) with 3 degrees of freedom and reduced integration. Each solid element had a size of $1 \mathrm{~mm}$, which was proven efficient and adequately accurate for such simple geometries. The finite element meshing, and boundary conditions are depicted in figure 1. 


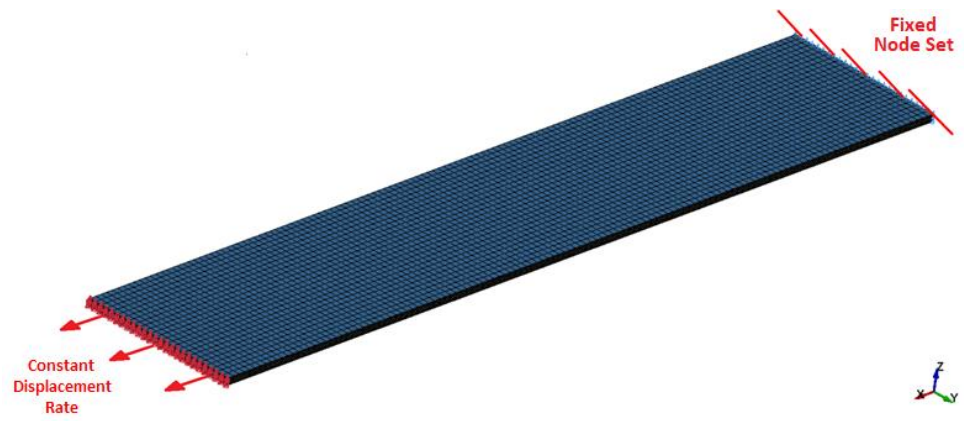

Fig. 1. Typical FE mesh and boundary conditions of the quasi-static tensile coupon

Simple tensile loading boundary conditions were implemented to the simulation, with one edge fixed in all 3 degrees of freedom and the other end displaced in a constant rate (100 $\mathrm{mm} / \mathrm{s}$ ) along the longitudinal axis of the laminate. Furthermore, the global damping parameter was set to 800 , while standard LS-Dyna hourglass formulation was used with a coefficient of 0.1 .

\subsection{Material damage models:}

The LS-Dyna material damage models chosen for the simulation are capable of composite material damage modeling [7]. Each model is based on different failure criteria and their accuracy of thermoplastic composite behavior simulation is evaluated based on experimental data found in [6].

\subsubsection{MAT_055}

This model is based on Tsai-Wu criteria. Matrix and fiber tensile and compressive modes are taken into consideration. The failure occurs when the following are fulfilled:

Tensile fiber mode:

$$
\begin{gathered}
\varepsilon_{f}^{2}=\left(\frac{\sigma_{\alpha \alpha}}{x t}\right)^{2}+\beta\left(\frac{\sigma_{\alpha b}}{s c}\right)^{2}-1 \\
\varepsilon_{f}^{2} \geq 0: \text { failed, } \quad \varepsilon_{f}^{2}<0 \text { : elastic behavior } \\
\text { when, } \quad E_{a}=E_{b}=G_{a b}=v_{b a}=v_{a b}=0
\end{gathered}
$$

Compressive fiber mode :

$$
\begin{gathered}
\varepsilon_{c}{ }^{2}=\left(\frac{\sigma_{\alpha \alpha}}{X c}\right)^{2}-1 \\
\varepsilon_{c}{ }^{2} \geq 0: \text { failed, } \quad \varepsilon_{c}{ }^{2}<0 \text { : elastic behavior } \\
\text { when, } \quad E_{a}=v_{b a}=v_{a b}=0
\end{gathered}
$$

Tensile and compressive matrix mode :

$$
\begin{gathered}
\varepsilon_{m d^{2}}{ }^{2}=\frac{\sigma_{b b}{ }^{2}}{Y c Y t}+\left(\frac{\sigma_{\alpha b}}{S c}\right)^{2}+\frac{(Y c-Y t) \sigma_{b b}}{Y c Y t}-1 \\
\varepsilon_{m d^{2}} \geq 0: \text { failed } \\
\varepsilon_{m d^{2}}<0: \text { elastic behavior }
\end{gathered}
$$




\subsubsection{MAT_162}

This material type is used to model the progressive failure analysis for composite materials of both unidirectional and woven layers. MAT_162 offers more advanced modeling to MAT_055 as it can simulate fiber, matrix failure and delamination under all loading conditions. It also incorporates the ability to simulate strain rate depended material behavior. The main failure and element erosion criteria for the unidirectional lamina model are listed below:

Tensile/shear fiber failure:

$$
f_{1}=\left(\frac{\left\langle\sigma_{\alpha}\right\rangle}{S_{b T}}\right)+\left(\frac{\tau^{2}{ }_{\alpha b}+\tau^{2} c a}{S^{2} F S}\right)-1=0
$$

Compression fiber failure:

$$
\begin{gathered}
f_{2}=\left(\frac{\left\langle\sigma_{\alpha}{ }_{\alpha}\right\rangle}{s_{a c}}\right)^{2}-1=0 \\
\text { where, } \quad \sigma_{\alpha}^{\prime}{ }_{\alpha}=-\sigma_{\alpha}+\left\langle\frac{\sigma_{b}+\sigma_{c}}{2}\right\rangle
\end{gathered}
$$

Perpendicular matrix mode:

$$
\begin{aligned}
& f_{4}=\left(\frac{\left\langle\sigma_{b}\right\rangle}{S_{b T}}\right)^{2}+\left(\frac{\tau_{b c}}{S_{b c}}\right)^{2}+\left(\frac{\tau_{a b}}{S_{a b}}\right)^{2}-1=0 \\
& \text { where, } \quad S_{b c}^{\prime}{ }_{b c}=S^{(0)} c a+\tan (\varphi)\left\langle-\sigma_{b}\right\rangle
\end{aligned}
$$

Parallel matrix mode (Delamination) :

$$
\begin{aligned}
& f_{5}=S^{2}\left\{\left(\frac{\left(\sigma_{c}\right)}{S_{b T}}\right)^{2}+\left(\frac{\tau_{b c}}{S{ }^{\prime \prime} b c}\right)^{2}+\left(\frac{\tau_{c a}}{S_{c a}}\right)^{2}\right\}-1=0 \\
& \text { where, } \quad S^{\prime \prime}{ }_{b c}=S^{(0)}{ }_{c a}+\tan (\varphi)\left\langle-\sigma_{c}\right\rangle
\end{aligned}
$$

\subsubsection{MAT_215}

This is a micromechanical model where the two phases (matrix \& fiber) are distinguished. It was designed specifically for the simulation of thermoplastic composite materials. Short fiber inclusions as well as unidirectional laminates can be modeled.

The inelastic tensile response of the matrix is taken under consideration via the stresseffective strain curve, where is demanded by the LS-Dyna material model card as input. Moreover, the mechanical properties of both the fibers and the matrix are required for the simulation.

Failure is considered either from a ductile damage initiation and evolution model for the matrix, or from a maximum stress criterion for the fibers.

\subsubsection{MAT_261}

This material model is capable of simulating fiber - reinforced composites by the continuum damage modeling technique. Non-linear in-plane shear mode can be modeled while fiber tension, compression are the other two modes considered.

Longitudinal (fiber) tension:

$$
f_{a}=\frac{\sigma_{a}}{X_{T}}=1
$$


Longitudinal (fiber) compression:

$$
f_{\text {kink }}=\left\{\begin{array}{l}
\left(\frac{\tau_{T}}{S_{T}-\mu_{T} \sigma_{n}}\right)^{2}+\left(\frac{\tau_{L}}{S_{L}-\mu_{L} \sigma_{n}}\right)^{2}=1, \text { if } \sigma_{b^{m}} \leq 0 \\
\left(\frac{\sigma_{n}}{Y_{T}}\right)^{2}+\left(\frac{\tau_{T}}{S_{T}}\right)^{2}+\left(\frac{\tau_{L}}{S_{L}}\right)^{2}=1, \text { if } \sigma_{b^{m}}>0
\end{array}\right.
$$

with: $S_{T}=\frac{Y_{C}}{2 \tan \varphi_{0}}, \quad \mu_{T}=-\frac{1}{\tan \varphi_{0}}, \quad \mu_{L}=S_{L} \frac{\mu_{T}}{S_{T}}$,

$$
\begin{gathered}
\sigma_{n}=\frac{\sigma_{b^{m}+} \sigma_{c^{\psi}}}{2}+\frac{\sigma_{b^{m}} \sigma_{c^{\psi}}}{2} \cos 2 \varphi+\tau_{b^{m} c^{\psi}} \sin 2 \varphi \\
\tau_{T}=-\frac{\sigma_{b^{m}-} \sigma_{c^{\psi}}}{2} \sin 2 \varphi+\tau_{b^{m} c^{\psi}} \cos 2 \varphi \\
\tau_{L}=\tau_{\alpha^{m} b^{\psi}} \cos \varphi+\tau_{c^{\psi} \alpha^{m}} \sin \varphi
\end{gathered}
$$

Transverse (matrix) failure:

$$
\begin{gathered}
f_{\text {mat }}=\left(\frac{\sigma_{n}}{Y_{T}}\right)^{2}+\left(\frac{\tau_{T}}{s_{T}}\right)^{2}+\left(\frac{\tau_{L}}{s_{L}}\right)^{2}=1, \text { if } \sigma_{n} \geq 0 \\
\text { with, } \quad \sigma_{n}=\frac{\sigma_{b+} \sigma_{c}}{2}+\frac{\sigma_{b-} \sigma_{c}}{2} \cos 2 \varphi+\tau_{b c} \sin 2 \varphi \\
\tau_{T}=-\frac{\sigma_{b-} \sigma_{c}}{2} \sin 2 \varphi+\tau_{b c} \cos 2 \varphi \\
\tau_{T}=\tau_{\alpha b} \cos \varphi+\tau_{c a} \sin \varphi
\end{gathered}
$$

Transverse (matrix) failure: transverse compression/shear

$$
f_{\text {mat }}=\left(\frac{\tau_{T}}{s_{T}-\mu_{T} \sigma_{n}}\right)^{2}+\left(\frac{\tau_{L}}{s_{L}-\mu_{L} \sigma_{n}}\right)^{2}=1,\left(\text { if } \sigma_{\alpha}<0\right)
$$

\section{Numerical simulation results}

The mechanical response of the quasi-static tensile loading analyses is depicted in figure 2 . The load-displacement curves from the 4 material damage models are compared to the reference curve found in [6].

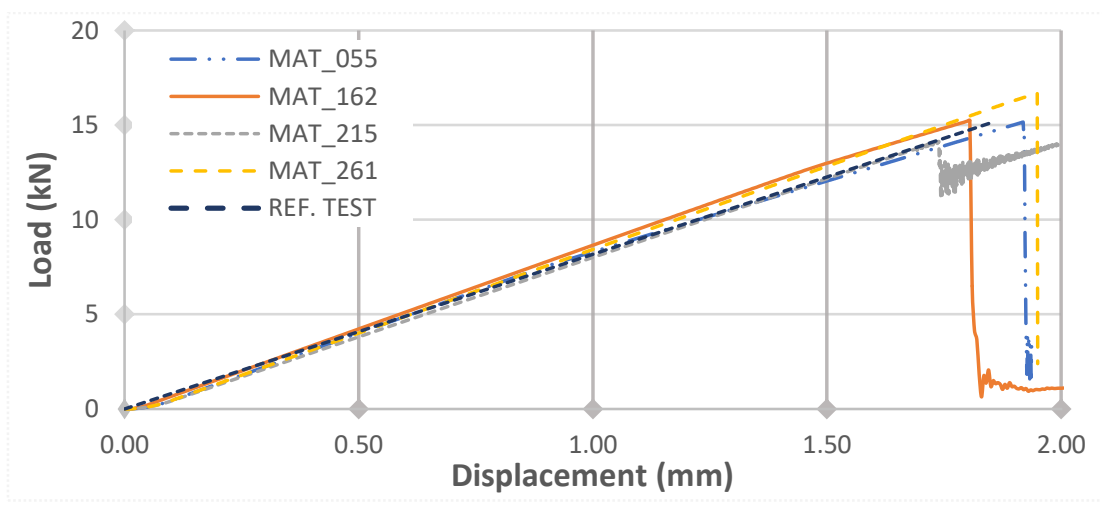

Fig. 2. Load-Displacement curves of the 4 material damage models and the reference test. 
The response of all the analyses was linear, as expected from the quasi-isotropic stacking sequence of the laminate. It is worth mentioning that the curve slopes of MAT_215 and MAT_055 almost coincide with the refence one. This indicates the fact that the stiffness and the behavior of the material under investigation was properly modeled. Furthermore, material models MAT_162 and MAT_055 stand closer to the reference test in terms of tensile strength prediction.

In reference to the failure sequence between the laminate plies, only MAT_162 and MAT_261 offers the ability to visualize the type of interlaminar or intralaminar degradation. Table 1 encapsules the results of the comparative study for the layers' failure and sequence observed in MAT_162 analysis at different load percentages. MAT_162 which has an integrated parallel matrix mode failure prediction feature didn't show delamination between the plies, which agrees with the reference experimental findings. The contours illustrate the damage accumulation in the laminate. Specifically, history variable \#10 indicates transverse matrix damage, while history variable \#7 shows fiber damage.

Table 1. Failure sequence comparison between reference experiment material models.

\begin{tabular}{|c|c|c|}
\hline Ref. Test [6] & MAT_162 & DAMAGE CONTOURS \\
\hline $\begin{array}{l}\text { Matrix cracks at }-45 \text { and } \\
90 \text { plies from } \\
\text { manufacturing } \\
\text { (0\% Load) }\end{array}$ & $\begin{array}{l}\text { Matrix cracking at } 45 \text { plies } \\
\text { (boundary plies) } \\
\text { (55\% Load) }\end{array}$ & 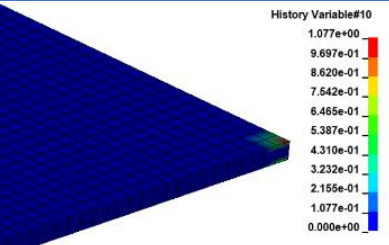 \\
\hline $\begin{array}{l}\text { Initial cracks propagation } \\
\text { at fiber rich regions } \\
(\mathbf{5 0 \%} \text { Load) }\end{array}$ & $\begin{array}{l}\text { Matrix cracking at - } \\
\text { 45plies adjacent to } 90 \\
\text { plies } \\
\text { (75\% Load) }\end{array}$ & 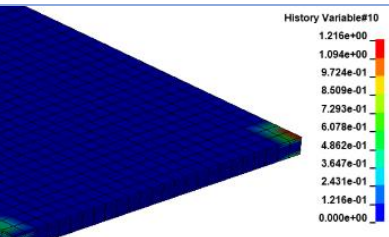 \\
\hline $\begin{array}{c}\text { New cracks at } 90 \text { plies and } \\
\pm 45 \text { plies and existing } \\
\text { crack propagation } \\
(\mathbf{8 0 - 1 0 0 \% ~ L o a d ) ~}\end{array}$ & $\begin{array}{l}\text { Crack propagation } \\
\text { (75-100\% Load) }\end{array}$ & 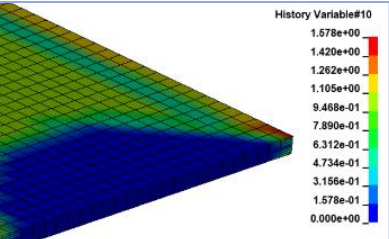 \\
\hline $\begin{array}{c}\text { Fiber breakage } \\
\text { (100\% Load - Failure) }\end{array}$ & $\begin{array}{c}\text { Fiber breakage } \\
\text { (100\% Load - Failure) }\end{array}$ & 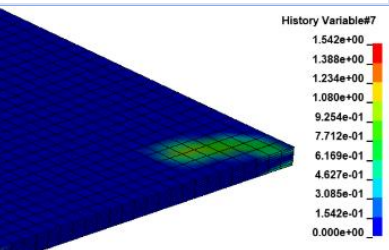 \\
\hline
\end{tabular}

\section{Conclusions}

In terms of mechanical response, the material model MAT_055 seemed to approach the behavior of the quasi-isotropic laminate quite satisfactorily. In combination with the limited user input needed it constitutes a capable material model for simple thermoplastic composite damage behavior simulations. 
MAT_162 presented both good approximation of the response and the failure sequence prediction. Given its ability to simulate delamination without the need of an extra CZM layer between plies, it is considered the most suitable material model for thermoplastic composite damage modeling.

MAT_215 can be used as an option when short fiber composites or more complex non quasi-isotropic composites need to be modeled. However, its micromechanical nature requires accurate input for each phase, making it more difficult to be handled by the user.

MAT_261 can be proven useful as a secondary solution to MAT_162, for complex thermoplastic composite simulations. It offers both response and failure sequence prediction with less demanding user input.

The material models can be ranked based on three different criteria. Table 2 visualizes results from personal experience, from most to least efficient.

Table 2: Material damage models ranking based on three criteria

\begin{tabular}{|c|c|c|}
\hline Mechanical Response & $\begin{array}{c}\text { Failure Sequence } \\
\text { Prediction }\end{array}$ & $\begin{array}{c}\text { Simplicity / User Input } \\
\text { MAT_055 }\end{array}$ \\
\hline MAT_215 & MAT_162 & MAT_055 \\
\hline MAT_162 & MAT_261 & MAT_261 \\
\hline MAT_261 & MAT_215 \\
\hline
\end{tabular}

\section{Acknowledgment}

The present study was conducted in the frame of the European aerospace project TORNADO Clean Sky 2 "Innovative Disbond Arrest Features for Long Thermoplastic Welded Joints".

TM: GKN Fokker, Partners: RESCOLL, KVE Composites Group, University of Patras

\section{References}

[1] R. Stewart, Thermoplastic composites - Recyclable and fast to process, Reinf. Plast. 55 (2011) 22-28. https://doi.org/10.1016/S0034-3617(11)70073-X.

[2] Y. Yang, R. Boom, B. Irion, D.J. van Heerden, P. Kuiper, H. de Wit, Recycling of composite materials, Chem. Eng. Process. Process Intensif. 51 (2012) 53-68. https://doi.org/10.1016/j.cep.2011.09.007.

[3] B. Galindo, R. Ruiz, G. Ulldemolins, C. Losada, Development of long fiber thermoplastic composites for industrial applications, Reinf. Plast. xxx (2021) 1-5. https://doi.org/10.1016/j.repl.2021.02.001.

[4] J.P. Reis, M. de Moura, S. Samborski, Thermoplastic composites and their promising applications in joining and repair composites structures: A review, Materials (Basel). 13 (2020) 1-33. https://doi.org/10.3390/ma13245832.

[5] H. Nishida, V. Carvelli, T. Fujii, K. Okubo, Thermoplastic vs. thermoset epoxy carbon textile composites, IOP Conf. Ser. Mater. Sci. Eng. 406 (2018). https://doi.org/10.1088/1757$\underline{899 X / 406 / 1 / 012043}$.

[6] M. Mohd Tahir, W.X. Wang, T. Matsubara, Failure behavior of quasi-isotropic carbon fiberreinforced polyamide composites under tension, Adv. Compos. Mater. 27 (2018) 483-497. https://doi.org/10.1080/09243046.2017.1405605.

[7] P. Reithofer, A. Fertschej, B. Hirschmann, B.J. Gmbh, Theory and Application notes, (2018). 